\title{
Efficient and Low Complexity Optimized Feature Spectrum Sensing with Receiver Offsets
}

\author{
Ikedieze Gabriel Anyim, John Chiverton, Misha Filip and Abdulkarim Tawfik. \\ University of Portsmouth, United Kingdom. \\ Email: up376711@myport.ac.uk \\ Abstract
}

\begin{abstract}
Spectrum awareness is an important function in the context of cognitive radio systems. It determines the presence or absence of free channels in the spectrum and identifies free channels for secondary users. Cyclostationary Feature Detection is an example of a spectrum awareness technique which involves the detection of signals based on their features such as cyclic frequencies, symbol rates, carrier frequencies and modulation types. It detects signals at very low signal-to-noise ratios. However there are performance degrading constraints such as cyclic and sampling clock offsets that can occur at the receiver end. These offsets result from local oscillator frequency offsets, Doppler effects and jitter. We propose an efficient low complexity multi-slot cyclostationary feature detector that reduces the effects of these constraints through an offline optimization approach that produces the number and size of slot and fast Fourier transform to be used. These slots and fast Fourier transforms are used to show the reduction of these offsets and the detection performance compared for the case of different signal to noise ratios in the presence or absence of the receiver offsets. Also, the complexity of the model is compared with the complexity of the conventional implementation and it shows significant reductions in the number of required computations.
\end{abstract}

Keywords — cognitive radio, spectrum sensing, cyclostationary, low complexity, optimization.

\section{INTRODUCTION}

Cognitive Radio (CR) can adapt to its surrounding communication environment and has emerged as a promising solution to address the problem of spectrum scarcity [1] by exploring spectral opportunities and deliver a more efficient utilization of the available spectral resources [2]. The CR concept can be divided into four main functional areas namely: (A) "Spectrum sensing" which determines the spectrum availability and the presence or absence of licensed primary users. (B) "Spectrum management" which predicts the duration that the spectrum holes (unused bandwidths) will be available for the secondary users before it is released again to the primary users. (C) "Spectrum sharing" for allocating the spectrum holes among the secondary users according to demand. (D) "Spectrum mobility" which maintains hitless or error free (seamless) communication during frequency allocation between the primary and secondary users and thereby produces better spectrum transition [3]. This research is on Spectrum sensing of the CR. Some researched spectrum awareness systems such as Energy Detection and Matched Filters are not capable of detecting signals at low Signal to Noise Ratio (SNR) in order to provide dynamic and accurate information to the secondary users in [3], [4]. The use of time-domain coprime sampling was adopted in [5]. This relies on the sub-bin energy detection of power spectral density (PSD) obtained from Fourier transform of estimated autocorrelation of the samples obtained from time-based coprime sampling. On the other hand a Cyclostationary Feature Detector (CFD) detects signals at low level SNRs through features of modulated signals such as spectrum and cyclic frequencies. It can also be implemented in a wideband scenario to reflect the real world communications environment. This requires the sampling of received signals at very high rates usually exceeding 1 Giga symbols per second (GSps), which is difficult to realize by state of art analogue to digital converters (ADC)s as discussed in [6].

Some authors have looked at wideband cyclostationary feature detector as in [4] where Welch Periodogram was set to produce mean square output of the signal which is equivalent to its energy and [7] which considered cooperative CR networks with prior knowledge of the primary user signal using generalized likelihood ratio detector. The strength in this depends on the uncertain effectiveness cooperation among the users. Spectral Analysis of Random Sampling on Grid (RSG) in wideband was investigated in [8] which utilizes sampling rate lower than the conventional Nyquist rate. This approach is exposed to undersamplng which will impact the correct detection of all the signals in the band especially under receiver constraints.

An approach discussed in [9] combined CFD with Compressive Signal Processing (CSP) without the need for signal reconstruction. However, the complexity of this combination was not elaborated. Recent works as in [10], adopted Complex valued Power Spectrum Density (CPSD) to detect the wideband signal using sub-Nyquist rate. It considers the signal as a complex value rather than as a magnitude as in Energy Detection. It was not clear how this method will deal a with noise-like Spread Spectrum signal or receiver offsets. Some studies were carried out on the reduction of the impacts of CFO on the CFD using the Cyclic Autocorrelation Function (CAF) for analysis as discussed in [11]. The use of the spectral correlation function (SCF) and fast Fourier transform (FFT) to reduce the effects of CFO and SCO has not been fully explored. Analysis in the frequency domain with FFT is less complex and computationally efficient as compared with time domain based analysis [12] and hence it is the adopted approach of this research to use SCF and FFT to reduce the effects of CFO and SCO.

In this paper, we propose a multi-slot wideband cyclostationary feature detector statistical model that uses the spectral correlation function (SCF) and multiple window-based short-time Fast Fourier Transforms (FFT)s to reduce the effects of receiver constraints namely: sampling clock offset (SCO) and cyclic frequency offset (CFO). The rest of this paper is divided into 3 sections as follows in section (II-A) where the conventional principles of SCF are formulated in terms of a CFD which are included in simulations to demonstrate the effects of SCO and CFO on the received signal. In section (III), 
the proposed system model was introduced and analyzed highlighting the offsets. This was followed by more simulations in section (IV) to arrive at the required slot and FFT sizes and numbers to reduce the effects of the offsets.

\section{Cyclostationary Feature Detection}

The fundamental principles used for feature detection along with the receiver constraints that affect the performance of the CFD will be discussed.

\section{A. Spectral Correlation Function}

The Autocorrelation Function (AF) of a signal $x(t)$ as a function of time $t$ with period $T$ is the similarity of a function with itself at time lag $\tau$. A process is said to show cyclostationarity if its AF and mean are periodic. As discussed in [13], [14], since the AF is periodic, it can be represented by Fourier series as:

$$
R_{x}\left(t+\frac{\tau}{2}, t-\frac{\tau}{2}\right)=\sum_{\alpha} R_{x}^{\alpha}(\tau) e^{j 2 \pi \alpha t},
$$

where $\alpha$ is the cyclic frequency at which the cyclostationary feature or second order periodicity of the modulated signal occurs. $R_{x}^{\alpha}(\tau)$ are the Fourier series coefficients and give the generalized Cyclic Auto-correlation Function (CAF),

$$
R_{x}^{\alpha}(\tau)=\frac{1}{T} \int_{-T / 2}^{T / 2} R_{x}\left(t+\frac{\tau}{2}, t-\frac{\tau}{2}\right) e^{-j 2 \pi \alpha t} \mathrm{~d} t .
$$

This can be expressed as using the time based signal $x(t)$ and its conjugate $x^{*}(t)$ as,

$$
R_{x}^{\alpha}(\tau)=\lim _{T \rightarrow \infty} \frac{1}{T} \int_{T} x\left(t+\frac{\tau}{2}\right) x^{*}\left(t-\frac{\tau}{2}\right) e^{-j 2 \pi \alpha t} \mathrm{~d} t
$$

where $T$ is the fundamental period and for a modulated signal it is a function of the symbol period and carrier frequency as in [13]. When $\alpha$ is zero, (3) gives the conventional Cyclic Autocorrelation Function (CAF) whose Fourier transform (FT) is the conventional Spectral Correlation Function (SCF) for the observation time $T^{\prime}$ and expressed as,

$$
S_{x}^{\alpha}(f)=\lim _{1 / T^{\prime} \rightarrow 0} \lim _{T \rightarrow \infty} \frac{1}{T^{\prime}} \int_{-T^{\prime} / 2}^{T^{\prime} / 2} \frac{1}{T} X_{T}\left(t, f+\frac{\alpha}{2}\right)
$$

$$
\times X_{T}^{*}\left(t, f-\frac{\alpha}{2}\right) \mathrm{d} t
$$

where $X_{T}(t, f)$ is the complex envelope of the narrow-band spectral component or short-time Fourier Transform of $x(t)$ with centre frequency $f$, period $T$ (FT length), bandwidth $1 / T$ (of the FT) and cyclic frequency $1 / T^{\prime}$. Further expressed as,

$$
X_{T}(t, f)=\int_{t-T / 2}^{t+T / 2} x(v) e^{-j 2 \pi f v} d v .
$$

Given $N^{\prime}$ number of samples, the SCF (4) can be represented discretely with,

$$
S_{x}^{\alpha}(n, f)=\frac{1}{N^{\prime}} \sum_{n=0}^{N^{\prime}-1} \frac{1}{L} X_{L}\left(l, f+\frac{\alpha}{2}\right) X_{L}^{*}\left(l, f-\frac{\alpha}{2}\right)
$$

as discussed in [15], where $N^{\prime}$ is the fixed sensing time, $X_{L}(l, f)$ is the $L$-point fast Fourier transform (FFT) around the $n^{\text {th }}$ sample with $L$ as the discrete FFT length. We refer to (6) as the conventional discrete Spectral Correlation Function (SCF). Typically, a Cyclostationary Feature Detector (CFD) uses either the CAF or SCF in the time or frequency domains respectively to detect cyclic features of the received modulated signal such as cyclic and spectrum frequencies as discussed in [15], [16].

\section{B. Effect of Cyclic Frequency and Sampling Clock}

Offsets on Spectral Correlation Function

Cyclic frequency $\alpha$ is dependent on both the symbol rate $1 / T$ and the carrier frequency $f_{c}$, where $T$ is the symbol period set by the clock at the transmitter [17]. Every clock produces some errors and in a local oscillator this results in frequency offsets as discussed in [17]. Also some uncertainties in the cyclic frequency will be introduced by Doppler shift. Cyclostationary Feature Detector (CFD) requires knowledge of the cyclic frequency and symbol rate to correctly detect the signals. Therefore, sub-optimal knowledge of cyclic frequency, symbol rate and carrier frequency results in cyclic frequency offset (CFO) at the receiver and this affects the detection performance of CFD and more significantly as the number of samples are increased as discussed in [11], [17]. Let the CFO be represented by $\Delta_{\alpha}$. Given that,

$$
\alpha^{\prime}=\alpha \times\left(1+\Delta_{\alpha}\right)
$$

where $\alpha^{\prime}$ and $\alpha$ are the actual and ideal cyclic frequencies (CF) at the Receiver and transmitter respectively. Some modulated signals have cyclic frequencies at both symbol rate and carrier frequency. The effect of CFO can be determined by substituting (7) for $\alpha$ in SCF (4).

Sampling Clock Offset (SCO) is another condition that affects feature detection. It occurs from the frequency offset produced by oscillators and insufficient knowledge of the symbol rate at the Analogue to Digital (A/D) stage of the 

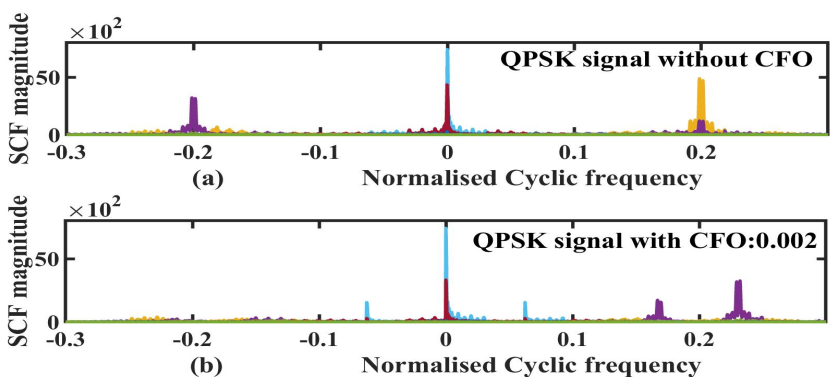

Fig. 1: The Effects of Receiver Offsets on the location of the QPSK signal. (a) SCF of QPSK signal without CFO. SCF of QPSK signal with CFO.

receiver. Sampling frequencies are produced by these oscillators, with $\mathrm{SCO} \delta$, and results in a drift in sampling times and this time-shift varies as the number of samples increases [11] producing phase shifts in the SCF estimate when the FFT bins are correlated. Fig. 1(a) shows in a frequency domain, the SCF of QPSK signal without any CFO. Fig. 1(b) shows how the cyclic frequency is being shifted in position as a result of the presence of CFO which will affect the possibility of correctly sensing the primary user's (PU) signal. SCO can be stated as in (8),

$$
T_{s}^{\prime}=(1+\delta) \times T_{s}
$$

where $T_{s}^{\prime}$ is the actual sampling period used at the receiver, $T_{s}$ is the ideal sampling period with good knowledge of the symbol rate at the transmitter and $\delta$ is the SCO. In order to adequately represent a signal, the sampling rate $f_{s}$ should be in multiples of the symbol rate $1 / T_{\text {symbol }}$, where $T_{\text {symbol }}$ is the symbol period. The objective of the model is to be robust to these effects so as to improve the possibility of detection through a statistical model using the SCF and FFT-based slot in a wideband scenario.

\section{SYSTEM MODEL}

We propose a wideband multi-slot window-based FFT statistical Test based CFD model that detects signals $x(t)$ by correlating in the frequency domain with the spectral correlation function (SCF) defined in (4) and (6). It covers the possibilities of reducing the effects of $\mathrm{CFO}$ and SCO. The received high frequency wideband signal is first down-converted to an Intermediate Frequency (IF) to the range of Mega-Hertz (MHz) and sampled as a baseband signal before correlation is computed. Given a wideband of $N_{B}$ samples divided into $N$-point slots the relationship can be stated as,

$$
P=\frac{N_{B}}{N}
$$

where $P$ and $N$ are the number of slots and samples per slot respectively. Each of the $P$ slots is of length $T=N T_{s}$ seconds, where $T_{s}$ is the nominal sampling period. Since we are detecting across the entire wideband, $T_{s}$ is applicable to the wideband being detected rather than the individual signal bandwidth of interest. This is different from a wideband approach where the $T_{s}$ applies to the signals of interest because in real world scenarios, all the signals may not be known and therefore makes implementation more complex. Our approach is also different from multi-channel wideband where each channel is sampled with a different oscillator which is not cost effective. The use of a window in the FFT computation reduces spectral leakage as the wideband is sampled. Applying a sliding window Fast Fourier Transform (FFT) to the computation of the complex envelope in (5) for the $p^{\text {th }}$ slot gives,

$$
X_{L}(n, k)=\sum_{r=-L / 2}^{+L / 2} w(r) x_{p}(n-r) e^{-j 2 \pi k(n-r) T_{s}}
$$

where $w(r)$ is a data tapering window of length $L$ of the narrow-band spectral component of the received signal $x_{p}(t)$ for the $p^{t h}$ slot. Considering a multi-slot wideband having the same slot size, the Test Statistic (TS) without the receiver constraints can be expressed as,

$$
S_{x}^{\alpha}(k)=\frac{1}{P} \sum_{p=1}^{P} S_{x^{*}}^{\alpha}(k, p)
$$

where $S_{x}^{\alpha}(k, p)$ is discrete SCF for the $p^{t h}$ slot of $N$ samples. Further expressed as,

$$
S_{x}^{\alpha}(k, p)=\frac{1}{N} \sum_{n=0}^{N-1} \frac{1}{L} X_{L, p}\left(n, k+\frac{\alpha}{2}\right) X_{L, p}^{*}\left(n, k-\frac{\alpha}{2}\right)
$$


where $X_{L, p}(n, k)$ is the $L$-point window-based FFT of the $p^{t h}$ slot around the $n^{t h}$ sample. Therefore, from the TS (11), the constrained Test Statistic $T S_{1}$ (Test Statistic with receiver offsets) can be written as,

$$
S_{x}^{\alpha^{\prime}}(k)=\frac{1}{P} \sum_{p=1}^{P}\left(\frac{1}{N} \sum_{n=0}^{N-1} \frac{1}{L} X_{L, p}\left(n, k+\frac{\alpha^{\prime}}{2}\right) \quad \times X_{L, p}^{*}\left(n, k-\frac{\alpha^{\prime}}{2}\right)\right)
$$

where $\alpha^{\prime}$ is the cyclic frequency affected by the CFO (7), while $P$ is the number of slots of $N$ size for the wideband over which the SCF is averaged. The TS is used to detect the features at signal's symbol rate $1 / T$ and carrier frequency (fc) as in Table I.

TABLE I: Cyclic features for some modulation types [9], [15], [18]

\begin{tabular}{|l|l|}
\hline Modulation type & Peaks at $(\alpha, f)$ \\
\hline BPSK & $\left(\frac{1}{T}, f_{c}\right),\left(2 f_{c}, 0\right),\left(2 f_{c} \pm \frac{1}{T}, 0\right)$ \\
\hline QPSK & $\left(\frac{1}{T}, f_{c}\right),\left(2 f_{c} \pm \frac{1}{T}, 0\right)$ \\
\hline MSK & $\left(\frac{1}{T}, f_{c}\right),\left(2 f_{c} \pm \frac{1}{2 T}, 0\right)$ \\
\hline QAM & $\left(\frac{1}{T}, f_{c}\right)$ \\
\hline
\end{tabular}

Equation (13) show that the TS is the correlation of the FFT of the received signal with itself as discussed in [19] and in this research across the wideband on a slot basis. It should be noted that the conventional discrete SCF (6) is a form of the proposed model TS in (13) where $P=1$ and $N=N^{\prime}$.

\section{A. Threshold and Detection}

A binary decision rule of two hypotheses will be adopted in order to detect the signals.

- Hypothesis $0, H_{0}$ for noise only,

$$
H_{0}: s(t)=w(t)
$$

- Hypothesis $1, H_{1}$ for signal present,

$$
H_{1}: s(t)=x(t)+w(t)
$$

where $x(t)$ and $w(t)$ are signal only and Additive White Gaussian Noise (AWGN) respectively. Fundamentally, Spectral Correlation Function (SCF) or cyclic autocorrelation function (CAF) is expected to be flat in the presence of noise or exhibits a non-zero mean since noise is a wide-sense stationary process and has no cyclic correlation of any order. Also, noise will not be affected by the SCO or CFO due to its stationary property. From the Central limit theorem (CLT) as in [20], the SCF distribution is Gaussian. The threshold that is approximately at the noise level and gives Constant False Alarm Rate (CFAR) $T_{\mathrm{d}}$ during the detection is selected. The Test Statistic $T S_{1}$ can be compared against the detection threshold $T_{d}$ to determine the presence or absence of the signal [21] as:

- $T S_{1}<T_{d}$ signal is absent, $H_{0}$.

- $T S_{1} \geq T_{d}$ signal is present, $H_{1}$.

The mean of the Test Statistic [22] at $H_{1}$ and $H_{0}$ can be expressed as ,

$$
\mu_{1}=E\left[S_{x}^{\alpha \prime}(k)\right]
$$

and

$$
\mu_{w}=E\left[S_{w}^{\alpha \prime}(k)\right]
$$

respectively. While the variance at $H_{1}$ and $H_{0}$ can be expressed as,

and

$$
\sigma_{s}^{2}=E\left[\left|S_{x}^{\alpha \prime}(k)\right|^{2}\right]-\left|E\left[S_{x}^{\alpha \prime}(k)\right]\right|^{2}
$$

$$
\sigma_{w}^{2}=E\left[\left|S_{w}^{\alpha \prime}(k)\right|^{2}\right]-\left|E\left[S_{w}^{\alpha \prime}(k)\right]\right|^{2} .
$$

Therefore, the probabilities of false alarm $P_{\mathrm{fa}}$ and detection $P_{\mathrm{d}}$ [11], [22], [23] can be expressed as in (17) and (18) below,

$$
P_{\mathrm{fa}}\left(T_{\mathrm{d}}\right)=\operatorname{Prob}\left\{T S_{1}>T_{\mathrm{d}} \mid H_{0}\right\}=\mathbb{Q}\left(\frac{\mu_{w}}{\sqrt{\sigma_{w}^{2}} / 2}, \frac{T_{\mathrm{d}}}{\sqrt{\sigma_{w}^{2}} / 2}\right),
$$

where $\mathbb{Q}(),. \sigma_{w}^{2}$ and $\mu_{w}$ are the generalized Marcum-Q function, noise variance and the mean of $T S_{1}$ at $H_{0}$,

$$
P_{\mathrm{d}}\left(T_{\mathrm{d}}\right)=\operatorname{Prob}\left\{T S_{1}>T_{\mathrm{d}} \mid H_{1}\right\}=\mathbb{Q}\left(\frac{\mu_{s}}{\sqrt{\sigma_{s}^{2}} / 2}, \frac{T_{\mathrm{d}}}{\sqrt{\sigma_{s}^{2}} / 2}\right),
$$

where $\mu_{s}$ and $\sigma_{s}^{2}$ are the mean and variance of $T S_{1}$ at $H_{1}$. 

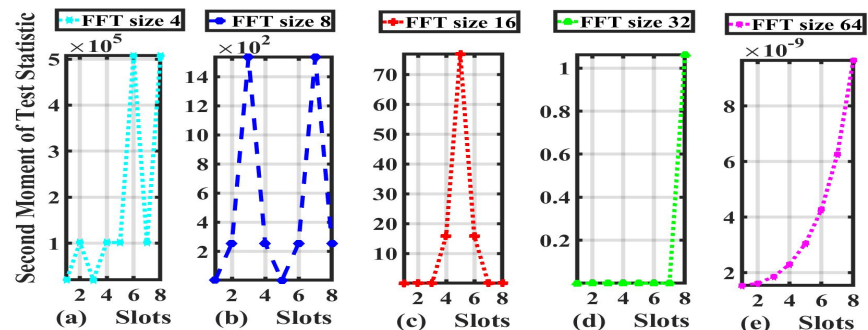

Fig. 2: Effects of different FFT sizes on the Second moment of the TS with $N=256$ and $P=8$.

\section{B. Computational Complexity}

Given the computational complexity $F F T_{c x}$ for 1 slot in the order of,

$$
O_{1}(M, L)=M\left(L \log _{2} L\right)
$$

where $L\left(\log _{2} L\right)$ is a single FFT complexity [15] and $M$ is the total number of FFTs in a slot. It is given that,

$$
M=\frac{N}{L}
$$

From (9), (22) and (23), we derive the total computational complexity $F F T_{t c}$ for the wideband in the order of,

$$
O_{T}(M, L)=P \times M \times L \log _{2} L
$$

As $N$ becomes smaller, more slots $P$ will be generated resulting in more FFTs $M$.

\section{Optimizing the FFT Size $L$ and Number $M$}

We will obtain the optimum $L$ and $M$ from the first optimization problem in (25) which will be used to further solve the second optimization (26) within the limitations of computational complexity and the minimum samples $L_{\min }$ required in a slot to represent the information. FFT size should be chosen for maximum Pd, correct frequency resolution and reduced complexity.

$$
(\bar{L}, \bar{M})=\underset{L, M}{\arg \max } P_{d}
$$

such that $L M \leq N$ and $L \geq L_{\min }$

where $\bar{L}$ and $\bar{M}$ are the optimized $L$ and $M$. From (9), (23) and (24) we can verify the effect of computational complexity.

\section{Optimizing the Slot Size $N$ and Number $P$}

As stated earlier, from (9), the number of slots $P$ is affected by the bandwidth $N_{B}$ or slot size $N$. Therefore, there is a need to optimize $N$ and $P$ for maximum $P_{d}$ for a given bandwidth. Also there is an additional limitation for $N$ in order to cover the minimum samples $N_{\text {min }}$ required for the information symbols within one slot. The optimization problem can be formulated from (9) and (21) as,

$$
(\bar{N}, \bar{P})=\underset{N, P}{\arg \max } P_{d}
$$

such that $N P=N_{B}$ and $N \leq N_{\min }$

where $\bar{N}$ and $\bar{P}$ are the optimized $N$ and $P$ and $P_{d}$ is the probability of detection. There will be an increase in $P$ for small $N$ as in (9) which will subsequently produce an increase in the number of FFTs which could impact on the computational complexity. In order to further solve the optimization problem in (26), consideration will be given to the overall complexity from combining multiple FFTs which is given in (24). The choice of $N$ and $P$ should be made for maximum Pd and reduced complexity. The optimization is applied in section (IV-C)

\section{RESULTS AND DISCUSSION}

\section{A. The effect of fast Fourier Transform size on the second moment of the Test Statistic}

Let us examine the effect of the Hanning window-based fast Fourier Transform (FFT) size $L$ on the second moment of the constrained Test statistic $T S_{1}$ (13) given as $\left|T S_{1}\right|^{2}$. For the purpose of correlation, the FFTs must be in the powers of 2 to benefit from the extra efficiency associated with FFT radix-2 routines. Given a bandwidth $B \mathrm{MHz}$, sampling rate $f_{s}=$ $4 \times B$ and assuming fixed $\mathrm{CFO}=\mathrm{SCO}$ at 1000 parts per million (ppm, $1 \times 10^{-3}$ ) and fixed sensing period $T$ of $1 \times 10^{-4}$ seconds for the wideband. For a $2.5 \mathrm{MHz}$ QPSK signal in a $5 \mathrm{MHz}$ bandwidth in Fig. 2, the use of different sizes of FFT produced correlation peaks specific to that FFT. It shows that the smaller the FFT, the higher the correlation envelopes. This is expected since more small FFTs are contained in any slot (23). Therefore, the smaller the FFT size the less the number of slots required to form the correlation peaks. The correlation magnitudes shown in all the plots for the magnitude of the second moment of the Test Statistic (TS) represent the expected spectral peaks due to the QPSK modulated signal as in Fig. 1. When the bandwidth is increased to $10 \mathrm{MHz}$ and the slot number $P$ is increased as shown in Fig. 3, the magnitude of 

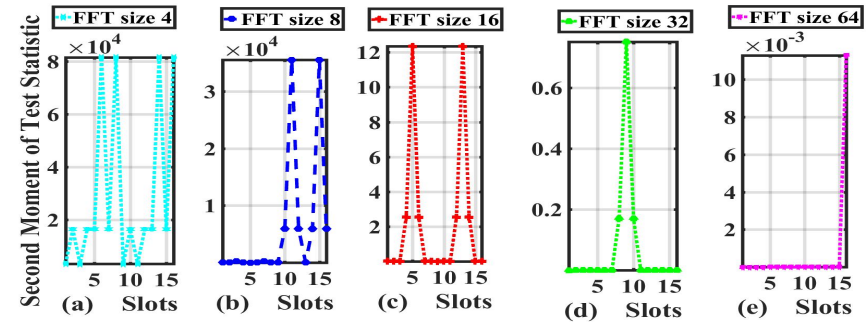

Fig. 3: Effects of FFT size on the TS Second moment with $\mathrm{N}=256$ and $\mathrm{P}=16$.
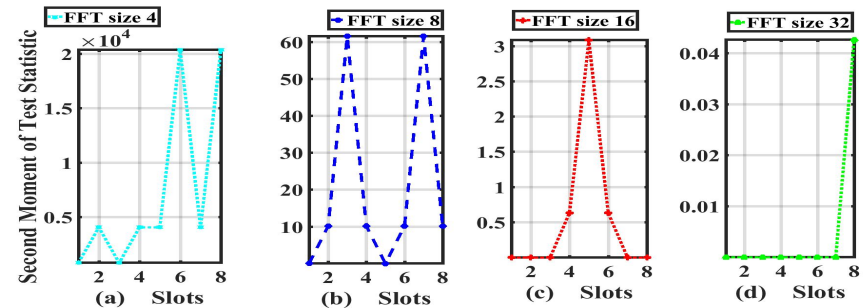

Fig. 4: Effects of FFT size on the TS Second moment with $\mathrm{N}=128$ and $\mathrm{P}=8$.
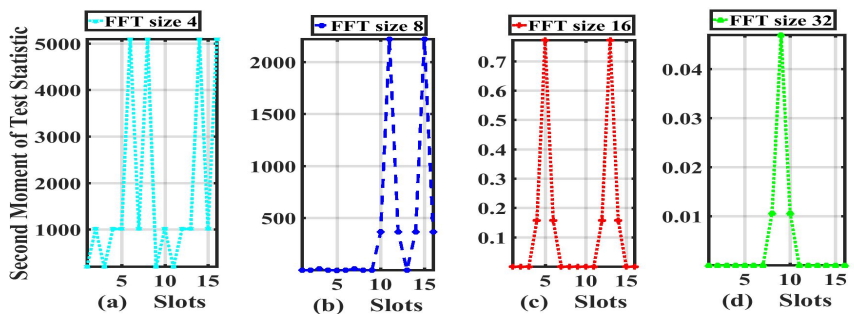

Fig. 5: Effects of FFT size on the TS Second moment with $\mathrm{N}=64$ and $\mathrm{P}=16$.

the second moment of $T S_{1}$ increased significantly for some FFTs. This is due to the increased sample size $N_{B}$ (4096) for the wideband (9). For instance, for FFT size $L=64$ samples, the magnitude of second moment of Test Statistic increased from $15 \times 10^{-9}$ to $10 \times 10^{-3}$ as in Fig. 3. It can be stated that because of the high peaks obtained with small FFTs, detection based on them will be more efficient as small narrowband spectral components will be correlated over the entire wideband. Furthermore, from Fig. 2 and Fig. 3, each FFT has a minimum number of slots for the correlation envelopes. For instance, FFT sizes 4, 8, 16 and 32 require 3, 5, 7 and 11 slots respectively. This is because the smaller the FFT size the smaller the narrowband spectral component that it can correlate which results in reduced samples. It can be stated that each FFT shows constant performance across different bandwidths with the smaller FFTs having higher magnitude of $T S$.

\section{B. The effect of slot size on the second moment of the Test Statistic}

We can reduce the slot size $N$ further to ascertain the effects on the constrained second moment of the TS. Consider a $2.5 \mathrm{MHz}$ frequency signal and $2.5 \mathrm{MHz}$ bandwidth (1024 samples) at fixed sensing period $T$ of $1 \times 10^{-4}$ seconds and $N$ reduced to 128 samples as in (4). It shows significant reductions in the magnitude of the TS second moment for each FFT. The reduction in magnitude is increased when $N$ is further reduced to 64 samples as in (5) using the same signal parameters. Considering Fig. 4 and Fig. 5 for the same bandwidth (1024 samples) and as the slot size is reduced (from 128 to 64 ) and the slot number increased, the magnitude of the correlation peaks decreases while the number of correlation peaks increases for each FFT size. This is due to the reduced number of FFTs in the slots (23). However, this decreasing magnitude of the peaks may affect the detection of the signal depending on the selected reference detection threshold. On the other hand, with the increased bandwidth (4096 samples) in Fig. 3 and compared with Fig. 4 for the same 16 slot numbers, the result is increased magnitude of the spectral peaks due to the increased number of FFTs (23) following the increase in $N$ to 512 in Fig. 4 as against 256 samples in Fig. 3. We can conclude that the FFT size and number of FFTs in a slot, $L$ and $M$ respectively determine the magnitude of the peaks. Although the combination of small slot and FFT sizes produce more correlation peaks and more efficient detection, the correlation peak magnitude should not fall below the selected reference threshold $T_{d}$. This is necessary in order to correctly detect the signal.

\section{Applying the Optimization}

The product of correlating the FFTs according to (13) is $L^{2}$ and gives the maximum computable slots $P$ for $L$ and the maximum $P$ for FFT lengths 4, 8 and 16 are 16, 64 and 256 respectively. According to the optimization requirements in (25), we should minimize $N$ which in turn creates more $P$. Therefore, the first concern in selecting an FFT is to consider the total $P$ that will cover the bandwidth to be sensed. Secondly, $N$ shall be selected to cover the certain minimum samples required by any $L$ to effectively correlate in (13). For instance FFT length 64 requires minimum of 256 samples to properly correlate. Given a bandwidth $N_{B}$ and $N$ of 4096 and 256 samples respectively as in (6), $P$ can be calculated from (9) as 4096/256 = 16 slots. From Fig. 6 FFT lengths 8 and 16 use the smallest sample sizes to reach the peak detection. Since we were interested in optimization, $N$ was further reduced to 64 and used with $L$ of 8 and 16 samples. Comparing $N$ 


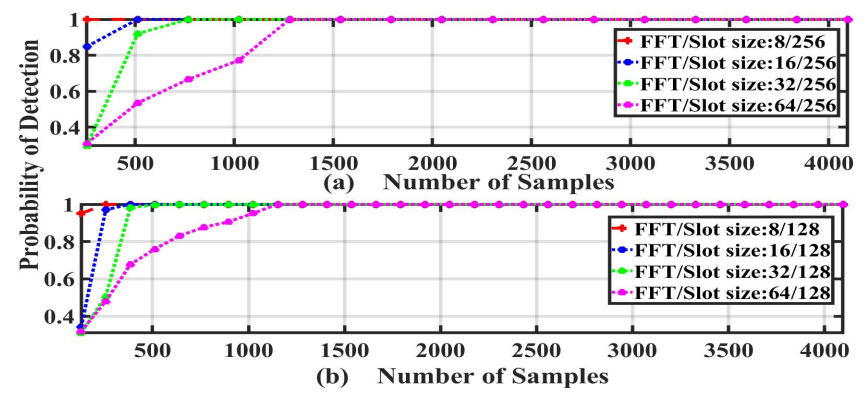

Fig. 6: Probability of detection for $10 \mathrm{MHz}$ bandwidth at $\mathrm{N}=256 / 128$ samples

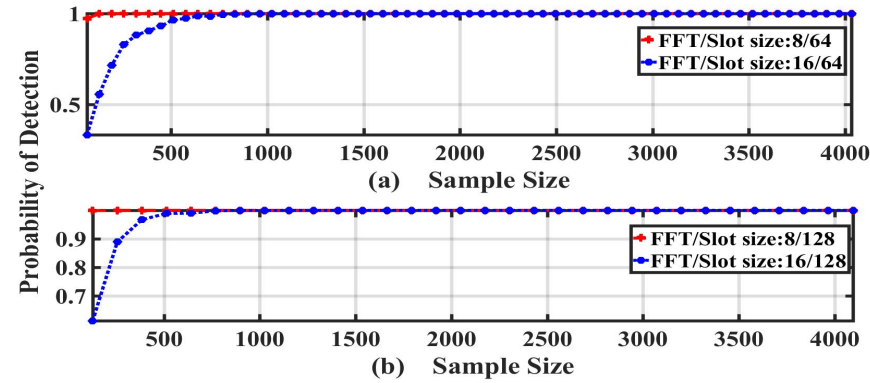

Fig. 7: Probability of detection for $10 \mathrm{MHz}$ bandwidth at $\mathrm{N}=64 / 128$ samples

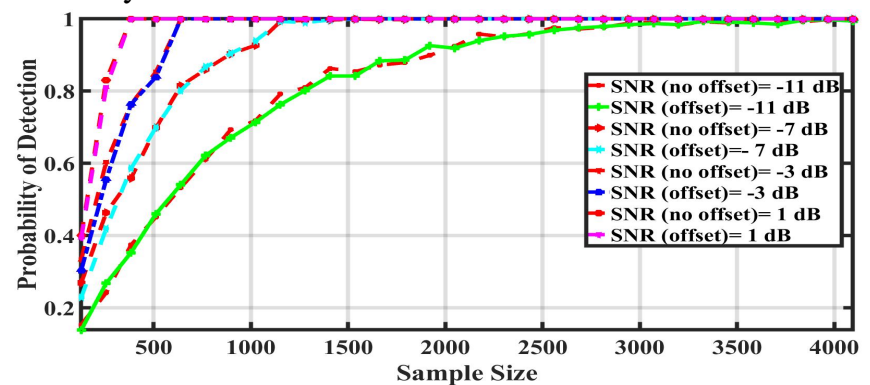

Fig. 8: Probability of detection with-without offsets, $10 \mathrm{MHz}$ BW at $\mathrm{N}=64$ and FFT $=16, \mathrm{CFO}=\mathrm{SCO}=1 \times 10^{-3}$.

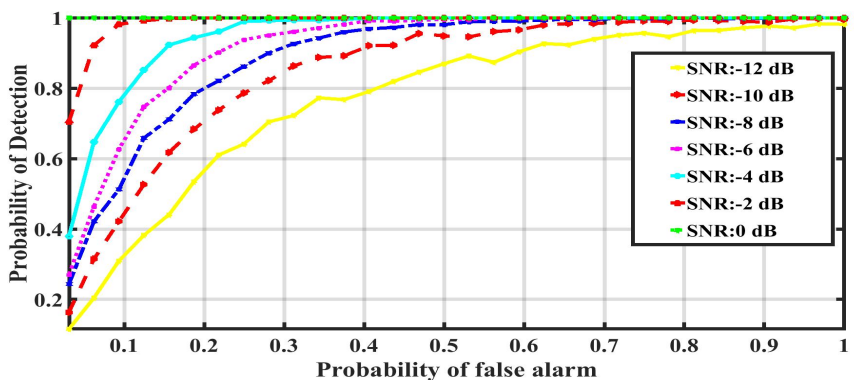

Fig. 9: Receiver Operating Characteristics at $10 \mathrm{MHz}$ signal, $\mathrm{N}=64$ and FFT $=16, \mathrm{CFO}=\mathrm{SCO}=1 \times 10^{-2}$

of 64 and 128 samples as in Fig. 7, the detection is better with $N$ of 64 samples for both FFT lengths. Apart from the maximum Pd requirement in (26), the frequency resolution and the resultant $P$ will also be considered in choosing an FFT size. FFT size 8 shows more smearing of signal energy across a wider frequency range which reduces the frequency resolution as shown by the closeness of the two peaks as in Fig. 7. Therefore, the optimized FFT size was selected to be 16. It should be noted that $N$ will not be reduced further for FFT size 8 due to the maximum computable slots $L^{2}$ already explained in this section; or for FFT size 16 due to the minimum $N$ required for acceptable correlation. With optimized values of $L$ and $N$ as 16 and 64 respectively, the obvious fact is that the number of slots $P$ have increased and the effect of this was then investigated with respect to the complexity described shortly. In summary, considering the simulations and calculations from (9) and (23), given a bandwidth (BW) of 4096 samples $N_{B}$, the optimized values of $L, N, P, M$ were found to be 16, 64, 64 and 4 (per slot) respectively giving 256 total FFTs. Therefore, we can obtain optimized values of $M, N, P$ for a selected small $L$. These optimized values were then used in comparing the performance of the model with and without receiver constraints at different values of SNR as in Fig. 8.

Observing the curves in Fig. 8, there is a closeness between a pair of curves for both with and without offsets obtained for each SNR. This shows that the effects of receiver offsets have been reduced by adopting small FFT and slot sizes. It also reduces the concern that large samples are needed for re-sampling for CFD under receiver impairments. Fig. (9) is the receiver operating characteristic (ROC) with the slot/FFT size pair $(64,16)$ under receiver offsets at different values of SNR. It shows the detection performance of the wideband cyclostationary feature detector with receiver constraints. In the presence of receiver constraints, low signal levels down to $-12 \mathrm{~dB}$ SNR were detectable by the constrained TS (13). The model detects much lower level signals under receiver constraints as shown in Fig. 8 and Fig. 9 when compared against 


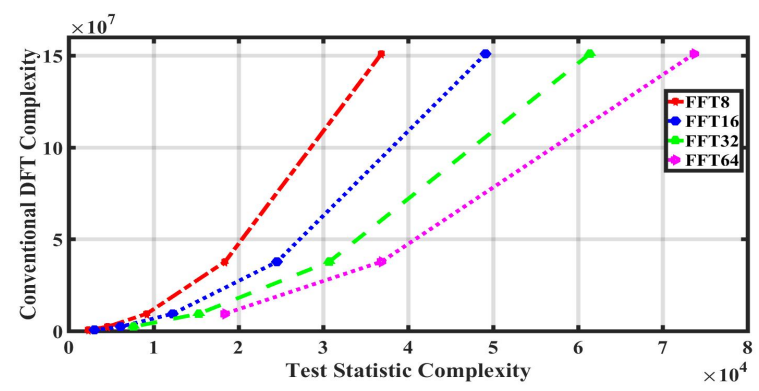

Fig. 10: Comparison between the complexities of Multi-slot Model Test Statistic and Conventional SCF based approach.

a time-based model discussed in [16]. It offers lower complexity, speed and accuracy due to the small size FFTs. The complexities of both the model Test Statistic (13) and the conventional SCF (6) were calculated according to (9), (22), (23), (24) and compared as in Fig. 10 for $N=256$ samples. It shows significant complexity gain.

\section{CONCLUSION}

In this paper, we have shown in the frequency domain that the effects of sampling clock and cyclic frequency offsets can be reduced by using the optimized sizes and numbers of slot size and fast Fourier transform for the implementation of wideband cyclostationary feature detector. It has been shown that the use of multi-slot and small FFTs do not increase the computational complexity of the model rather results in low complexity efficient detection process that is applicable to different bandwidths. This is in contrast to the approach of using large samples in terms of the cost and computational complexity. This model serves as a guide to the hardware design and implementation of wideband cyclostationary feature detectors under receiver constraints.

\section{REFERENCES}

[1] K. Patil, R. Prasad, and K. Skouby, "A survey of worldwide spectrum occupancy measurement campaigns for cognitive radio," in International conf. on devices and comms (ICDeCom), 2011, pp. 1-5.

[2] D. Tarchi and et al, "Technical challenges for cognitive radio application in satellite comms," in IEEE 9th International conf. on CR oriented wireless networks, Oulu, Finland, Jun. 2014, pp. 136-142.

[3] T. Yucek and H. Arslan, "A survey of spectrum sensing algorithms for cognitive radio applications," IEEE communications survey and tutorials, vol. 11, pp. 116-130, Mar. 2009.

[4] D. Cabric, S. M. Mishra, and R. W. Brodersen, "Implementation issues in spectrum sensing for CR," in IEEE 38th Asilomar Conf. Signals, Systems and Computers, Pacific Grove, USA, Mar. 2004, pp. 772-776.

[5] S. Ren, Z. Zeng, C. Guo, and X. Sun, "Wideband spectrum sensing based on coprime sampling," in IEEE 22nd International conf. on telecomms (ICT), Sydney, Australia, Mar. 2015, pp. 248-352.

[6] H. Sun, A. Nallanathan, C. Wang, and Y. Chen, "Wideband spectrum sensing for cognitive radio networks: a survey," IEEE Wireless Communications Magazine, vol. 20 no. 2, pp. 74-81, Apr. 2013.

[7] D. M. Najafabadi, A. A. Tadaion, and M. R. A. Sahaf, "Wideband spectrum sensing by compressed measurements," in IEEE Computers and Comms Symposium, Cappadocia, Turkey, Jul. 2012, pp. 667-671.

[8] B. I. Ahmad, A. Tarczynski, and A. Al-Ani, "A sars multi-band spect. sens. method in wideband comm. sys using RSG," in 19th European Conf. on Sig. Proc. Conf., Barcelona, Aug. 2011, pp. 1219-1223.

[9] M. Guo, F. Hu, Y. Wu, S. Kumar, and J. D. Matyjas, "Feature-based compressive signal processing CSP measurement design for the pattern analysis of CR spectrum," in IEEE Conf. on Global Comms. Conf. (GLOBECOM), Atlanta, GA, Dec. 2013, pp. 1131-1136.

[10] M. Damavandi and V. Nader-Esfahani, "Compressive wideband spectrum sensing in cognitive radio systems using CPSD," in IEEE International Conf. on Comms, Signal Processing, and their Applications (ICCSPA), Sharjah, Feb. 2015, pp. 1-6.

[11] E. Rebeiz, P. Urriza, and D. Cabric, "Optimizing wideband cyclostationary spectrum sensing under receiver impairments," IEEE Trans. on signal processing, vol. 61, No. 15, pp. 3931-3943, May 2013.

[12] R. S. Roberts, W. A. Brown, and H. H. Loomis, "Computationally efficient algorithms for cyclic spectral analysis," IEEE signal Processing Society, vol. 8, No. 2, pp. 28-49, Apr. 1991.

[13] W. A. Gardner, Introduction to random processes with applications to signals and systems. New York: McGraw-Hill, Inc, 1990.

[14] K. Kim and et al, "Cyclostationary approaches to signal detection and classification in cognitive radio," in IEEE New frontiers in dynamic spectrum access networks (DySPAN), Dublin, Apr. 2007, pp. 212-215.

[15] W. A. Gardner, "Signal interception: unifying theoretical framework for feature detection," IEEE Trans. on comms, vol. 36, No. 8, pp. 897-906, Aug. 1988.

[16] A. V. Dandawate and G. B. Giannakis, "Statistical tests for presence of cyclostationarity," IEEE Transactions on signal processing, vol. 42, No. 9, pp. 2355-2369, Sep. 1994.

[17] Y. Zeng and Y. Liang, "Robustness of the cyclostationary detection to cyclic frequency mismatch," in IEEE 21st International symposium on personal indoor and mobile radio comms (PIMRC), Istanbul, Sep. 2010, pp. 2704-2709.

[18] D. Cohen, E. Rebeiz, V. Jain, Y. C. Elder, and D. Cabric, "Cyclostationary feature detection from sub nyquist samples," in IEEE 4th International Workshop Computational Advances in Multi-Sensor Adaptive Processing (CAMSAP), San Juan, Dec. 2011, pp. 333-336.

[19] A. Tkachenko, D. Cabric, and R. W. Brodersen, "Cyclostationary feature detector experiments using reconfigurable bee2," in IEEE International Symposium on New Frontiers in Dynamic Spectrum Access Networks, Dublin, Ireland, Apr. 2007, pp. $216-219$.

[20] Z. Quan, S. Cui, H. V. Poor, and A. H. Sayed, "Collaborative wideband sensing for CR," IEEE Sig. Proc. Mag., vol. 25, no. 6, pp. 60-73, 2008.

[21] I. G. Anyim, J. Chiverton, A. Tawfik, and M. Filip, "The implementation of wideband cyclostationary feature detector with receiver constraints," in IEEE European Conf. on Networks and Comms. (EUCNC), Oulu, Finland, Jun. 2017, pp. 1-5.

[22] S. M. Ross, Introduction to probability models (9th ed.). New York: Academic Press, 2007.

[23] D. A. Shnidman, "The calculation of the probability of detection and the generalized marcum q-function," IEEE Trans. on Information Theory, vol. 35, no. 2, pp. 151-163, 1989. 